\title{
Plasma drift estimates from the Dynasonde: comparison with EISCAT measurements
}

\author{
K. J. F. Sedgemore ${ }^{1, *}$, J. W. Wright ${ }^{1}$, P. J. S. Williams ${ }^{1}$, G. O. L. Jones ${ }^{2}$, M. T. Rietveld ${ }^{3}$ \\ ${ }^{1}$ Prifysgol Cymru Aberystwyth, Ceredigion, SY23 3BZ, Wales \\ ${ }^{2}$ British Antarctic Survey, NERC, High Cross, Madingley Rd., Cambridge CB3 OET, England \\ ${ }^{3}$ EISCAT Scientific Association, Ramfjordmoen, N-9020 Tromsdaten, Norway \\ Received: 26 September 1997 / Revised: 9 January 1998 / Accepted: 16 January 1998
}

\begin{abstract}
Modern ionosondes make almost simultaneous measurements of the time rate of change of phase path in different directions and at different heights. By combining these 'Doppler' measurements and angles of arrival of many such radar echoes it is possible to derive reliable estimates of plasma drift velocity for a defined scattering volume. Results from both multifrequency and kinesonde-mode soundings at 3-min resolution show that the Dynasonde-derived $F$-region drift velocity is in good agreement with EISCAT, despite data loss during intervals of 'blanketing' by intense $E$-region ionisation. It is clear that the Tromsø Dynasonde, employing standard operating modes, gives a reliable indication of overall convection patterns during quiet to moderately active conditions.
\end{abstract}

Key words. Auroral ionosphere - Plasma convection · Instruments and techniques

\section{Introduction}

Ionosondes, operating near vertical incidence, have the potential to provide good temporal and spatial resolution at all latitudes. They are relatively inexpensive when compared with other ionospheric radar systems and are used extensively to monitor both long-term temporal and medium- to large-scale spatial variations in the atmosphere.

The use of modern ionosondes to estimate plasma drift is not new; the Doppler drift technique, using fixedfrequency soundings, was developed for the Digisonde (DGS) community in order to measure $F$-region plasma

Correspondence to: K. J. F. Sedgemore

*Present address: Dept. Physics, University of Southampton, Highfield, S017 1BJ, UK e-mail: kjs@soton.ac.uk flow in the polar cap (Buchau et al., 1988; Cannon et al., 1991). Verification of the Doppler drift technique by comparison with flow vectors derived from a co-located, beam-swinging incoherent-scatter radar (ISR) near Kangerlussuaq in Greenland shows mean DGS velocities to be within $50 \mathrm{~ms}^{-1}$ of ISR velocities, within the uncertainties of each instrument (Scali et al., 1995). Although the mean values correlate well there are a number of periods where marked discrepancies can be seen, especially in the magnetic north-south component. The authors attribute these discrepancies to the effects of horizontal gradients and temporal variations within the DGS sampling volume, aliasing in the frequency spectrum and beam-swinging errors in the ISR-derived velocities.

A similar technique, the VFIT technique, has been developed using an NOAA HF Radar operating as a Dynasonde (Grubb, 1979; Wright and Pitteway, 1979), the main difference being that multifrequency ionogram data are used rather than the two fixed frequencies of the Doppler drift technique. The VFIT (Wright and Pitteway, 1994) and Doppler drift techniques share nearly the same equations defining vector velocities from echolocation and line-of-sight Doppler measurements; the main differences are to be found at the level of data acquisition (e.g. Dynasonde 'echoes' and DGS 'sources'). Instead of using $\Delta \phi / \Delta t$ directly, VFIT scales the phase measurements into a velocity:

$V^{*}=\frac{c}{4 \pi f} \frac{\Delta \phi}{\Delta t}$,

where $c$ is the speed of light in vacuo, $f$ the radio frequency, $\phi$ the phase and $t$ time. This tends to weight the Doppler measurements in favour of moving targets and against $\Delta \phi / \Delta t$ arising from changes in underlying ionisation. Other and more subtle differences appear in data weighting, windowing, summarising and qualifying the results.

Initial results have been published by Jarvis (1995) of Dynasonde-derived velocities over Halley, Antarctica 
using a multifrequency technique similar to VFIT; these compare favourably with simultaneous plasma flow vectors measured poleward of Halley by the PACE coherent HF radar. A direct comparison of Dynasondederived drift measurements with EISCAT-derived velocity vectors by Sedgemore et al. (1996) showed good agreement for measurements taken during quiet to moderately active conditions, with the results averaged over time-scales of around $30 \mathrm{~min}$. In this paper we present results from an EISCAT comparison using an experimental Dynasonde pulse scheme which show good agreement on much shorter time-scales.

\section{Dynasonde velocity measurements}

Echoes are discriminated from impulsive noise in real time by a coincidence detection algorithm in the Dynasonde operating system (Wright and Pitteway, 1979; Rose, 1993); this is integrated with a multipulse scheme by which echo phases are measured in two receivers from a small six-antenna receiving array, at closely spaced times $(0.01 \mathrm{~s})$ and at radio frequencies differing by a few $\mathrm{kHz}$. Physical parameters are then derived by a UNIPHASE algorithm (Wright, 1988). A detailed discussion of multipulse schemes can be found in Pitteway and Wright (1922) and Tsai et al. (1993).

With the four-pulse schemes used previously, eight phases were available to determine six parameters: stationary-phase group range $R^{\prime}$, two components of echolocation $X L$ (east) and $Y L$ (north), echo 'Doppler velocity' $V^{*}$, polarisation, and an average phase angle. An estimation-error parameter $E P$, of two degrees of freedom, was also obtained. For the data presented here an experimental eight-pulse scheme was used instead. This doubles the time-span of the pulse set with immediate benefit to the Doppler resolution (Jarvis, 1995); with its revised $\Delta f$ pattern the Doppler aliasing interval is increased from $180^{\circ}$ to $360^{\circ}$, and $E P$ is estimated with ten degrees of freedom.

Individually for each echo, the line-of-sight velocity $V^{*}$ is related to the velocity components $V_{x}, V_{y}, V_{z}$ of a plane, totally reflecting surface located at $X L, Y L, Z L$, where $Z L^{2}=R^{\prime 2}-X L^{2}-Y L^{2}$, as:

$V^{*}=\frac{X L \cdot V_{x}+Y L \cdot V_{y}+Z L \cdot V_{z}}{R^{\prime}}$.

With a group of $N>3$ echoes, and the assumption that they share the same velocity vector, an estimate of the velocity vector is obtained by minimising:

$S=\sum_{i=1}^{N} w_{i}^{2} \cdot\left[V_{i}^{*}-\left(\frac{X L}{R^{\prime}} \cdot V_{x}+\frac{Y L}{R^{\prime}} \cdot V_{y}+\frac{Z L}{R^{\prime}} \cdot V_{z}\right)_{i}\right]^{2}$.

A weight $w$, varying inversely with $E P$, is assigned to each observation. The weighted, minimised variance $S$ is used together with diagonal elements of the covariance matrix to obtain confidence limits on the velocity components. $S$ is used also with the weighted $V^{*}$ variance $S Q=\sum_{i}\left(w_{i} \cdot V_{i}^{*}\right)^{2}$ to define $P V=1-S / S Q$ : the fraction of Doppler variance expressed by the solution. With $P V$ we can quantify the validity of the assumption that the $N$ echoes share the same velocity.

These steps do not in themselves lead us to favour any particular physical interpretation of the vector velocities obtained. Different interpretations, not many of which have been worked out in satisfactory detail, will apply in different situations. For example, in conditions where $V^{*}, X L$ and $Y L$ behave in a manner organised by a medium-scale Atmospheric Gravity Wave (AGW), the resulting vector velocities may describe phase-velocity components of the AGW (Wright and Pitteway, 1982). For the present work a simple model is implicit for the reflecting surface already mentioned. Specular reflection is assumed to occur from the plasma-density surface appropriate to the Dynasonde measurement frequency, and this surface is assumed to advect with the plasma itself. In common with the interpretation of EISCAT $F$-region velocities, velocity components normal to the magnetic field are assumed to be independent of height (above $160 \mathrm{~km}$ ) along the field line and imposed by a perpendicular electric field.

\section{Instrumentation}

The EISCAT experiment used in the following comparison is CP-1-K: a fixed, field-aligned beam with the remote sites intersecting at $278 \mathrm{~km}$ altitude. The velocity is actually determined for a single intersection height, but as the magnetic field lines are assumed to be at the same potential, each field-perpendicular component of plasma velocity should be almost constant over the whole of the $F$ region so that the comparison with Dynasonde velocities is valid at all heights.

The Dynasonde transmits $60-\mu$ s pulses at any frequency in the range $0.1-30 \mathrm{MHz}$ and two receivers are tuned electronically to the transmitted frequency. For the measurements described in this paper, signals were received using an array of six dipole antennas arranged in the shape of a cross (Pitteway and Wright, 1992). For this experiment, $B$-mode soundings, each lasting around $150 \mathrm{~s}$, were run every $3 \mathrm{~min}$ with a frequency range of $1-$ $10 \mathrm{MHz}$, and the pulse scheme used was one with eight pulses per set in place of the usual four. Along with the 3-min $B$-mode sounding schedule, single kinesondemode soundings ( $K$ mode) with four frequencies transmitted at 3.5, 4.0, 4.5 and $5.4 \mathrm{MHz}$, using the same eight-pulse scheme, were run every $30 \mathrm{~min}$. $K$ mode resembles in some ways the DGS drift mode and is intended for high-time-resolution studies (Wright and Pitteway, 1979).

\section{Velocity comparison}

The EISCAT data in the following comparison were taken from the Common Programme CP-1-K of 17-19 June 1996. In this experiment data dumps occur every $5 \mathrm{~s}$, although in this case the long-pulse raw data were 
post-integrated over a 3-min period in order to reduce the noise errors to a reasonable level, and also to match the cycle time for the Dynasonde soundings. The integrated data were then used to derive $F$-region vector velocities at $278 \mathrm{~km}$ altitude with the tristatic method. Dynasonde $B$-mode soundings were separated by 3 -min intervals, and all echoes with reflection frequencies between 2.8 and $8 \mathrm{MHz}$, virtual ranges between 200 and $500 \mathrm{~km}$, and angles of arrival within $20^{\circ}$ of the zenith were included in the analysis.

The eastward component of plasma velocity is shown in Fig. 1 at 3-min resolution. Looking first at the EISCAT velocities, a number of sudden westward surges can be seen at 1300, 1430 and 1600 UT, 17 June 1996, reaching a maximum of $600 \mathrm{~m} \mathrm{~s}^{-1}$. At 2045 UT the plasma begins to flow eastward during another active period seen as hard-precipitation events in the EISCAT electron-density data. After 2300 UT the flow rate decreases and remains stable at around zero for $14 \mathrm{~h}$, after which it turns westward again following the same pattern as on the previous day. Following the next flow reversal (Harang discontinuity) the eastward velocity increases to a maximum of $800 \mathrm{~m} \mathrm{~s}^{-1}$ during a highly active period. EISCAT noise errors in this component range from 60 to $90 \mathrm{~m} \mathrm{~s}^{-1}$ except during the final, disturbed period where they rise to around $120 \mathrm{~m} \mathrm{~s}^{-1}$. In comparison, the Dynasonde errors, derived as a measure of the goodness of the fit, are more variable ranging from 15 to $200 \mathrm{~m} \mathrm{~s}^{-1}$.

Throughout the whole experiment, lasting $45 \mathrm{~h}$ in all, there are sufficient Dynasonde echoes for the VFIT analysis, apart from two periods of 'blanketing' corresponding to the time of the major precipitation events seen in the magnetometer and EISCAT electron-density data. Blanketing occurs when an intense $E$ layer blocks out all, or nearly all $F$-region echoes. The magnetic $H$ component for 17 June indicated a number of disturbances, the first three of which correspond to the precipitation events seen during the first $6 \mathrm{~h}$ of the experiment. The fourth matches with the hard-precipitation event that occurred shortly before 2100 UT. The magnetogram for 18 June showed very little activity until around 2315 UT when a clear substorm signature could be seen with a 500-nT bay in the $H$ component.

After the first $3 \mathrm{~h}$ of small-amplitude velocities there is good agreement between EISCAT and Dynasonde $B$ mode velocities as the plasma flows westward, although the Dynasonde underestimates the peak magnitude at around 1600 UT. There is then an anomalous feature in the Dynasonde results seen as a $500 \mathrm{~m} \mathrm{~s}^{-1}$ westward excursion at 1815 UT during a period where EISCAT sees the plasma moving westwards at $300 \mathrm{~m} \mathrm{~s}^{-1}$. The ionograms from this time show a number of structured $E$-region echoes from within the Dynasonde sampling volume which may be the cause of this discrepancy.

At around 2100 UT there is a hard-precipitation event, and here the velocity fitting becomes impossible owing to insufficient echoes. The $F$-region signal returns at 2300 UT but with few echoes the velocity fits oscillate wildly for about $1 \mathrm{~h}$. From 0000 until 0200 UT, 18 June, there is a significant discrepancy between EISCAT and the Dynasonde which again may be due to the influence of $E$-region echoes in the sampling volume. From 0200 to $1400 \mathrm{UT}$, the ionosphere is quiet and inactive and the east-west velocity is effectively zero throughout this period. At 1400 UT there is a small, $150 \mathrm{~m} \mathrm{~s}^{-1}$ westward excursion lasting for $2 \mathrm{~h}$. The velocity then reverses for a short while before turning westward again reaching a maximum of $500 \mathrm{~m} \mathrm{~s}^{-1}$ at 1815 UT (Fig. 2). Throughout this time EISCAT and the Dynasonde are in close agreement, and this continues up to the Harang discontinuity and the onset of a major substorm at 2315 UT when blanketing occurs.

The $K$-mode velocities, shown also in Fig. 1, tend to follow the $B$-mode values. There is one interesting difference between 0000 and 0200 UT, 18 June. This corresponds to the period where there is a sustained discrepancy between EISCAT and the Dynasonde. It is
EISCAT \& Dynasonde (CP-1-K, 17-19 June 1996)

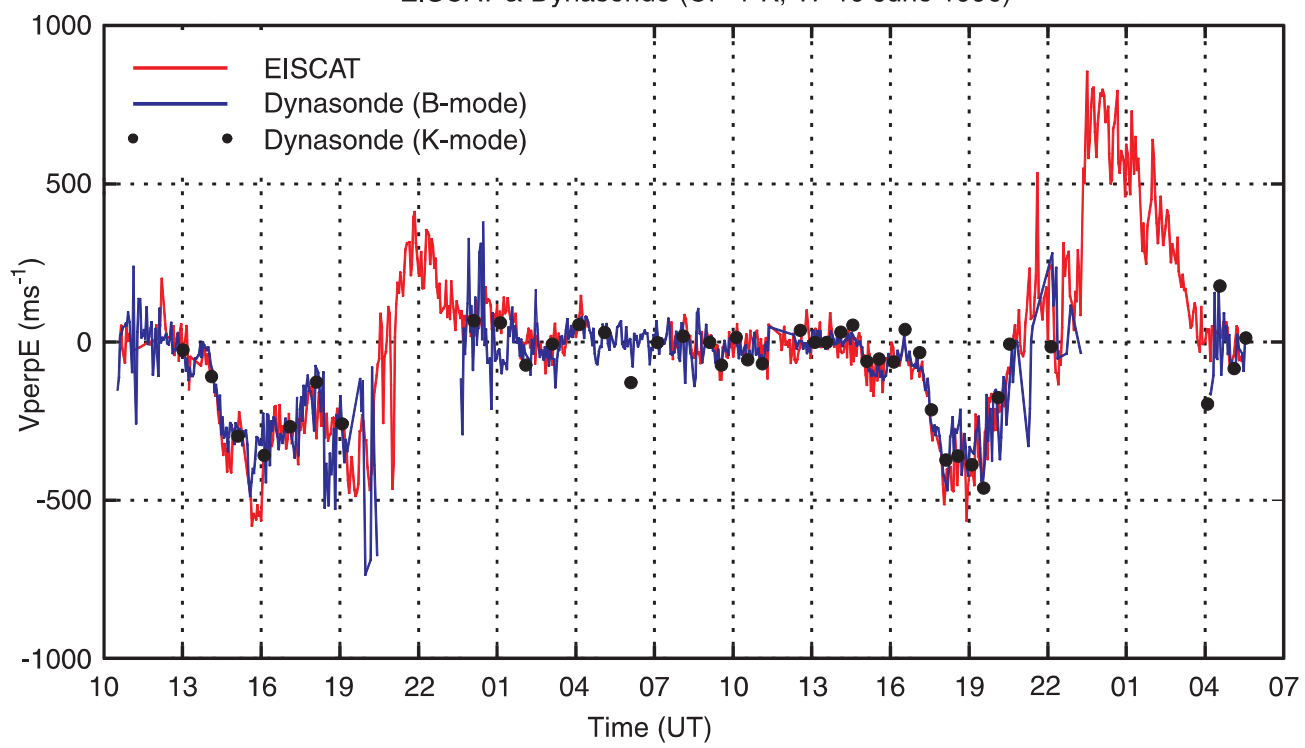

Fig. 1. EISCAT and Dynasonde $F$-region velocities (3-min resolution) from the experiment $\mathrm{CP}-1$ K, 17-19 June 1996. The eastward component perpendicular to the magnetic field is shown. EISCAT noise errors range from 60 to $120 \mathrm{~m} \mathrm{~s}^{-1}$, and Dynasonde errors from 15 to $200 \mathrm{~m} \mathrm{~s}^{-1}$. There is a gap in the EISCAT data between 0430 and 0730 UT, 18 June 


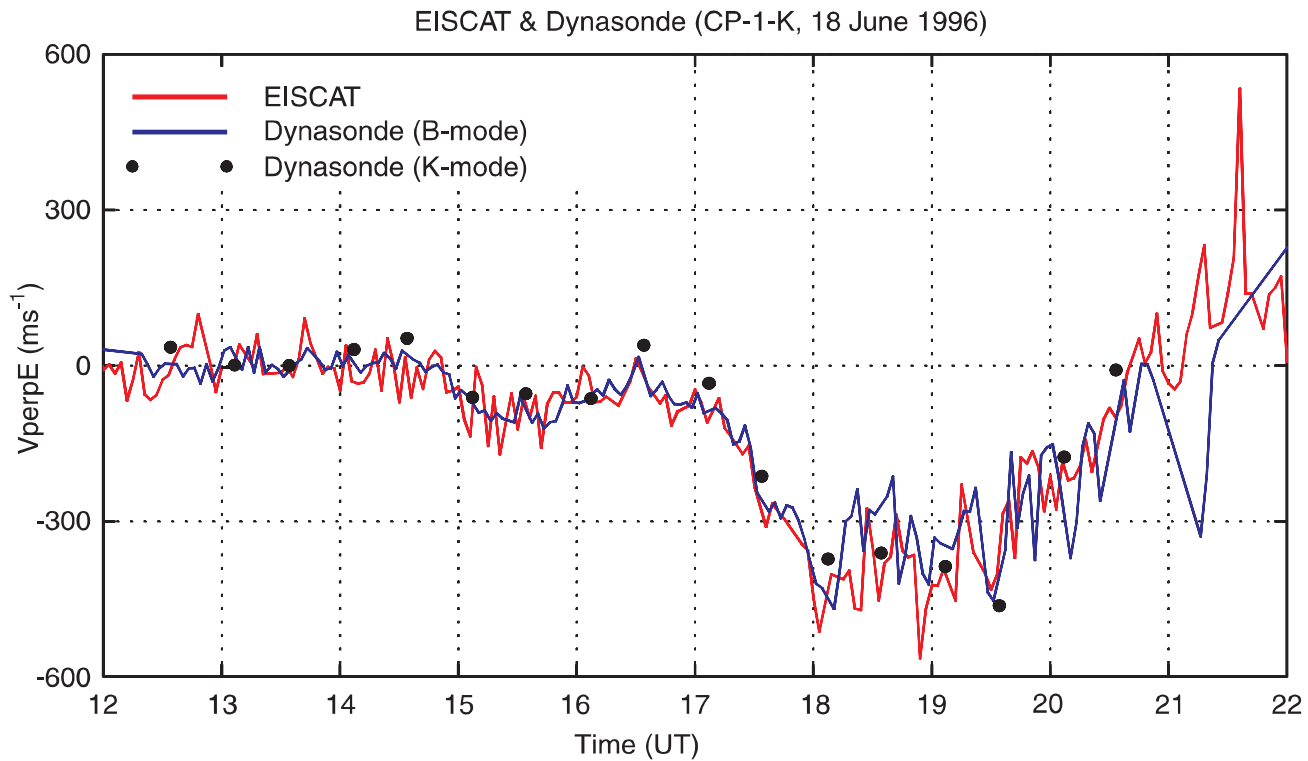

Fig. 2. EISCAT and Dynasonde $F$-region velocities (3-min resolution) from the experiment $\mathrm{CP}$ 1-K, 18 June 1996. The eastward component perpendicular to the magnetic field is shown, from 1200 to 2200 UT difficult to interpret the discrepancy between $B$ - and $K$ mode velocities at around 0630 UT (18 June) given the lack of EISCAT data at this time. Apart from these two instances the sounding modes give broadly similar results. A more rigorous test would involve an experiment which alternates $B$ - and $K$-mode soundings at 6min overall resolution. In general, however, the timeresolution advantage that $K$ mode has over ionogramtype soundings is outweighed by the far greater amount of information contained within $B$-mode soundings.

The magnetic north-south component of velocity (Fig. 3) displays little variation; the magnitudes involved are rather small and the error bars proportionately large. There is, however, one (southward) feature of peak magnitude around $100 \mathrm{~m} \mathrm{~s}^{-1}$ at around $1900 \mathrm{UT}$, 18 June, that stands out as significant given the EISCAT noise errors of $40-70 \mathrm{~m} \mathrm{~s}^{-1}$. Despite the small magnitudes and the scatter in Dynasonde velocities it is still possible to see a positive correlation between the two distributions.

This paper has concentrated on horizontal plasma drift. VFIT, however, may be used to produce a threedimensional vector fit. In this comparison the Dynasonde-derived field-aligned component is in good agreement with EISCAT, at around zero on average, but there are a small number of anomalously large values which could in future be used to identify 'bad' fits once an acceptable deviation from the mean was decided upon. It may be worth using such a restriction on the parallel component, although with the danger of rejecting genuinely large field-aligned flows. Another improvement to the technique would involve estimating

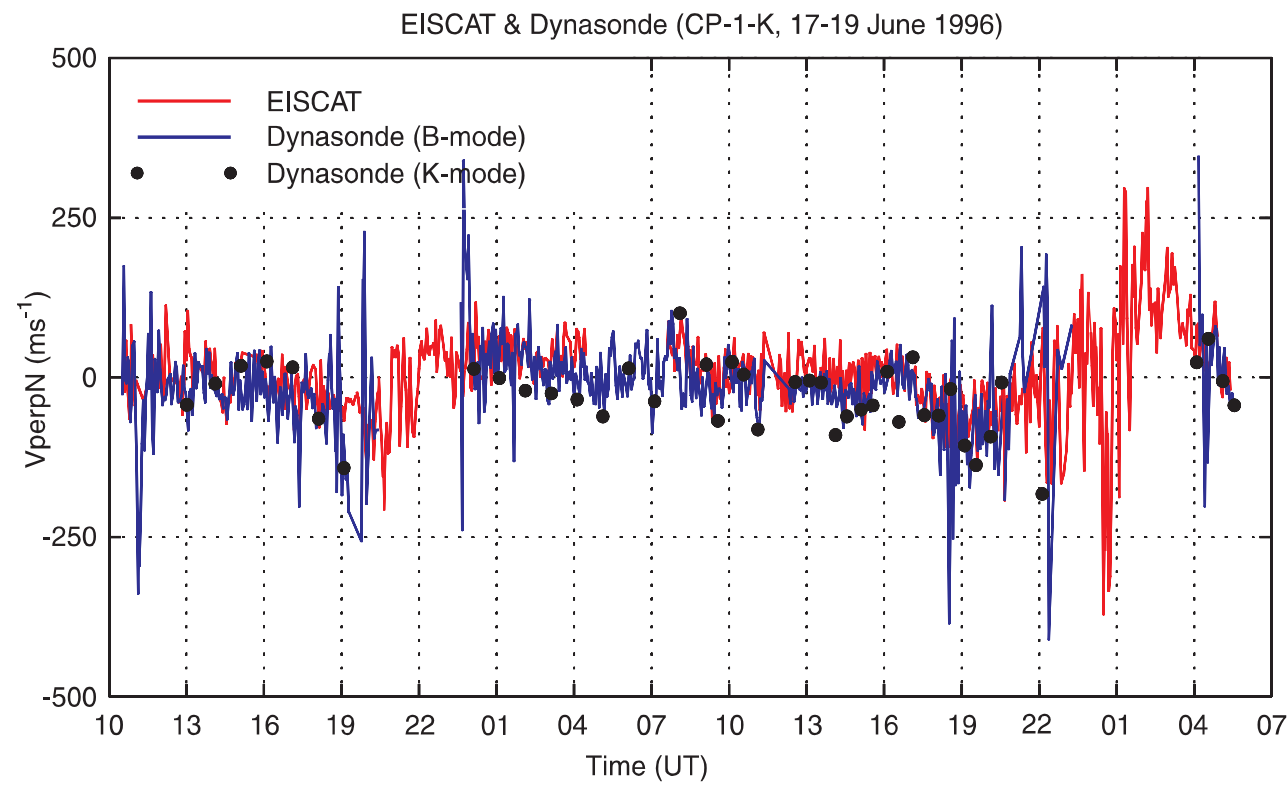

Fig. 3. EISCAT and Dynasonde $F$-region velocities (3-min resolution) from the experiment CP-1K, 17-19 June 1996. The northward component perpendicular to the magnetic field is shown. EISCAT noise errors range from 40 to $70 \mathrm{~m} \mathrm{~s}^{-1}$, and Dynasonde errors from 6 to $80 \mathrm{~m} \mathrm{~s}^{-1}$. There is a gap in the EISCAT data between 0430 and 0730 UT, 18 June 


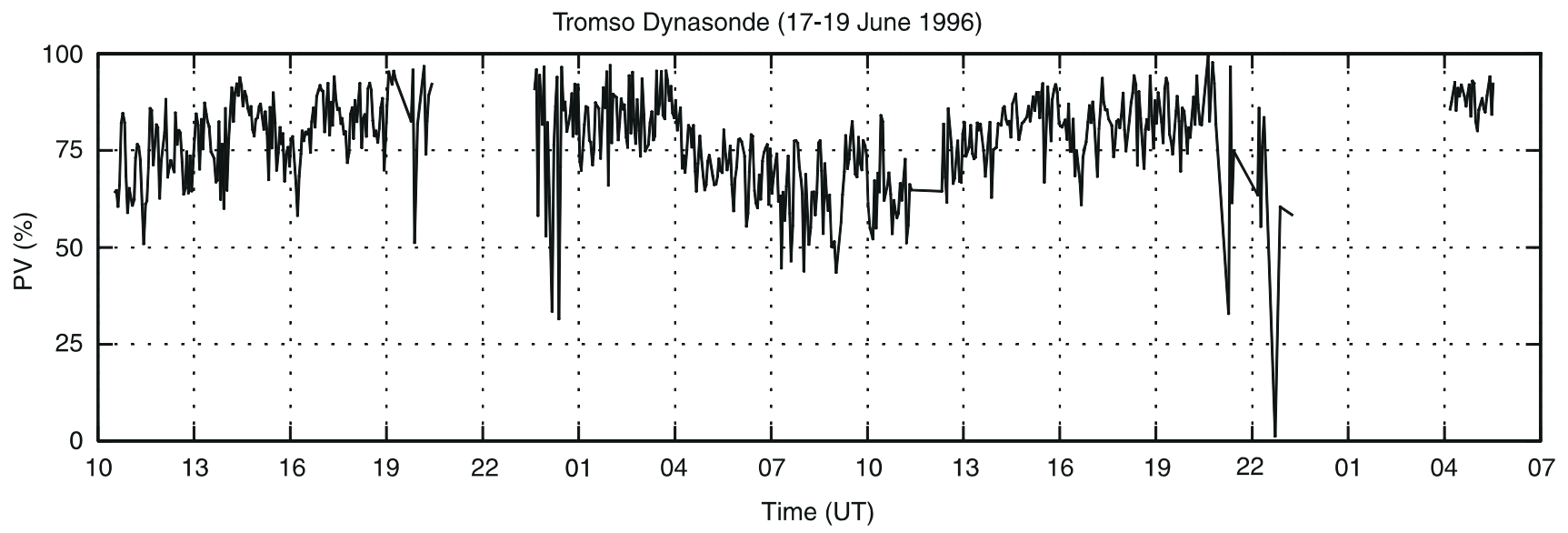

Fig. 4. Dynasonde percentage of Doppler variance $(P V)$ expressed by the vector velocities shown in Figs. 1 and 3, during the experiment $\mathrm{CP}-1-\mathrm{K}$, 17-19 June 1996
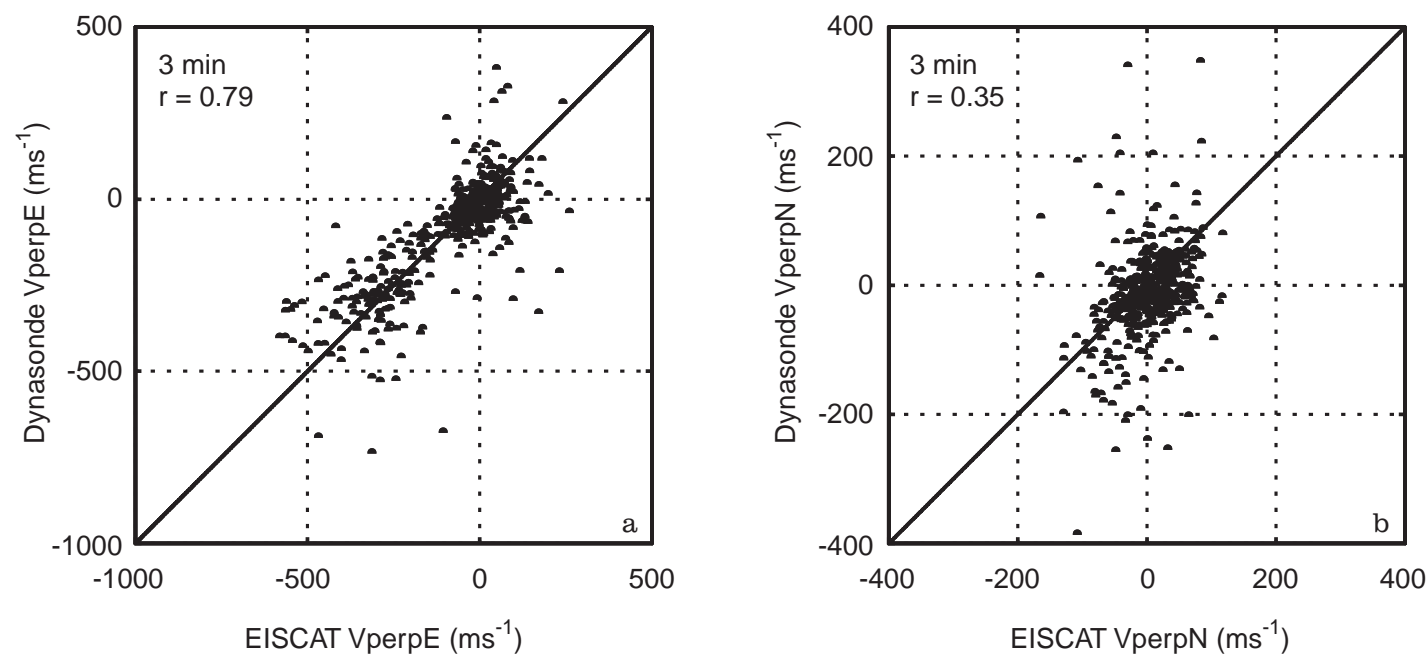

Fig. 5a, b. Correlation between EISCAT and Dynasonde F-region velocities (3-min resolution) during the experiment CP-1-K, 17-19 June 1996; $\mathbf{a}$ eastward and $\mathbf{b}$ northward components perpendicular to the magnetic field. In each case a line of unit slope is plotted for reference

horizontal gradients in velocity across the Dynasonde field of view by fitting velocity vectors to different quadrants.

Good agreement between EISCAT and Dynasonde $B$-mode velocities is reflected in the high $P V$ throughout the course of the experiment (Fig. 4). This seldom falls below $60 \%$ and during the periods of close agreement it is around $80 \%$. This is around $10 \%$ higher on average than for the four-pulse $B$-mode soundings used in the previous comparison. The few periods of low $P V$ correspond to the periods leading up to blanketing. In those cases where there is no tristatic ISR to provide a true value of plasma drift velocity, it is important to have some criterion derived from Dynasonde data which indicates whether the derived velocities are real; the evidence so far is that $P V$ provides just such a criterion.

When the correlation between the velocities from EISCAT and Dynasonde-derived data is calculated there is an approximately linear increase as the results are averaged over time-periods of 3,6,9 min and so on up to $54 \mathrm{~min}$. In the previous comparison by Sedgemore et al. (1996) it was necessary to average over periods of 30 min or more before a reasonably high correlation was obtained. With the present measurements of eastwest plasma drift, data with a time-resolution of $3 \mathrm{~min}$ give a correlation coefficient of 0.79 and this increases to 0.85 when the data are averaged over $54 \mathrm{~min}$. In the auroral zone north-south velocities are generally much smaller and hence the correlation is more seriously affected by random noise errors. Nevertheless, there is a 0.35 correlation between the north-south velocities measured with a 3-min resolution, and this increases to 0.50 for $54-\mathrm{min}$ averages. Figure 5 shows the correlation at 3-min resolution, for both the eastward and northward vector components.

\section{Conclusions}

The critical test for the Dynasonde is whether it can provide reliable values of plasma drift velocity in a stand-alone situation. This is particularly important at 
high latitudes where large and variable plasma drift occurs as a result of magnetospheric convection. For example, Dynasonde measurements of plasma drift above Longyearbyen could make a valuable contribution to the interpretation of data from the EISCAT Svalbard Radar (ESR), which at present is a monostatic system and hence cannot be used to measure velocities under conditions where rapid variations invalidate beam-swinging methods.

The first published comparison of EISCAT and Dynasonde velocities by Sedgemore et al. (1996) showed poor agreement for short-term variations but a high correlation between measurements of plasma velocity on time-scales of $30 \mathrm{~min}$ or more. The results presented here show that small-scale velocity variations can be resolved by the Dynasonde using standard operating modes at high time-resolution.

A major problem with using ionosondes to measure plasma velocity in the auroral zone is data gaps due to ratio absorption and intense $E$ layers which blanket echo returns from the $F$ region. In the polar cap these effects are far less common and velocities derived by Scali et al. (1995) using a DGS system based in Greenland show very few such gaps. This is a good argument for siting a Dynasonde next to the ESR, which for much of the time sits on the northern boundary of the auroral oval.

Acknowledgements. The authors are most grateful to the Director and Staff of EISCAT, and to the University of Tromsø for the data used in this work. EISCAT is an international facility supported by Finland, France, Germany, Japan, Norway, Sweden and the United Kingdom. During the period this work was undertaken KJFS was supported by a CASE award from the UK Particle Physics and Astronomy Research Council and the British Antarctic Survey.

Topical Editor D. Alcaydé thanks T. Berkey and J. Davies for their help in evaluating this paper.

\section{References}

Buchau, J., B. W. Reinisch, D. N. Anderson, E. J. Weber, and C. Dosois, Polar cap plasma convection measurements and their relevance to the modeling of the high-latitude ionosphere, Radio Sci., 23, 521-536, 1988.

Cannon, P. S., B. W. Reinisch, J. Buchau, and T. W. Bullett, Response of the polar cap $F$-region convection direction to changes in the interplanetary magnetic field: Digisonde measurements in northern Greenland, J. Geophys. Res., 96, 12391250, 1991.

Grubb, R. N., The NOAA SEL HF radar system (ionospheric sounder), Tech. memo, ERL SEL-55, NOAA SEL, 1979.

Jarvis, M. J., First Dynasonde observations of $F$-region plasma flow at Halley, Antarctica, J. Atmos. Terr. Phys., 57, 16111622, 1995.

Pitteway, M. L. V., and J. W. Wright, Toward an optimum receiving array and pulse set for the Dynasonde, Radio Sci., 27, 481-490, 1992.

Rose, M. C., FAIS User Manual, Tech. report, British Antarctic Survey, 1993.

Scali, J. L., B. W. Reinisch, C. J. Heinselman, and T. W. Bullet, Coordinated Digisonde and incoherent-scatter radar $F$-region drift measurements at Sondre Stromfjord, Radio Sci., 30, 14811498, 1995.

Sedgemore, K. J. F., P. J. S. Williams, G. O. L. Jones, and J. W. Wright, A comparison of EISCAT and Dynasonde measurements of the auroral ionosphere, Ann. Geophysicae, 14, 14031412, 1996.

Tsai, L.-C., F. T. Berkey, and G. S. Stiles, On the derivation of an improved parameter configuration for the Dynasonde, Radio Sci., 28, 785-793.

Wright, J. W., The Tromsø Dynasonde and its use as a correlative diagnostic with EISCAT, Tech. report, British Antarctic Survey, 1988.

Wright, J. W., and M. L. V. Pitteway, Real-time data acquisition and interpretation capabilities of the Dynasonde: 1. data acquisition and real-time display Radio Sci., 14, 815-825, 1979.

Wright, J. W., and M. L. V. Pitteway, Application of dopplionograms and gonionograms to atmospheric gravity-wave disturbances in the ionosphere, J. Geophys. Res., 87, 1719-1721, 1982.

Wright, J. W., and M. L. V. Pitteway, High-resolution vector velocity determinations from the Dynasonde, J. Atmos. Terr. Phys., 56, 961-977, 1994. 